\title{
Non conducting spherically symmetric fluids
}

\author{
Kayll Lake * \\ Department of Physics, Queen's University, Kingston, Ontario, Canada, K'7L 3N6
}

(Dated: November 6, 2018)

\begin{abstract}
A class of spherically symmetric spacetimes invariantly defined by a zero flux condition is examined first from a purely geometrical point of view and then physically by way of Einstein's equations for a general fluid decomposition of the energy-momentum tensor. The approach, which allows a formal inversion of Einstein's equations, explains, for example, why spherically symmetric perfect fluids with spatially homogeneous energy density must be shearfree.
\end{abstract}

Any spacetime is an "exact solution" Einstein's equations, but only those which are "physically reasonable" are worthy of consideration. Whereas the term "exact solution" has no precise definition, the concept is well understood 1] 2]. The concept of "physically reasonable" is somewhat more slippery and it is the responsibility of the author of any new exact solution to justify its significance. Clearly the force fitting of a metric to a sufficiently general energymomentum tensor is but nonsense. However, proceeding from a general energy-momentum tensor to more restricted cases can provide insight into properties of the idealizations. Here we consider spherically symmetric spacetimes which admit a fluid source subject to a zero flux condition equivalent to the requirement of zero thermal conductivity. First, we proceed in an invariant purely geometrical fashion. Then, by way of Einstein's equations, we translate our results into physical (albeit phenomenological) parameters.

Consider a spherically symmetric spacetime $\mathcal{M}[3]$

$$
d s_{\mathcal{M}}^{2}=d s_{\Sigma}^{2}+R^{2} d \Omega^{2}
$$

where $d \Omega^{2}$ is the metric of a unit sphere $\left(d \theta^{2}+\sin ^{2}(\theta) d \phi^{2}\right)$ and $R=R\left(x^{1}, x^{2}\right)$ where the coordinates on the Lorentzian two space $\Sigma$ are labelled as $x^{1}$ and $x^{2}$. (No specific choice of coordinates on $\Sigma$ will be made.) Consider a flow (a congruence of unit timelike vectors $u^{\alpha}$ ) tangent to an open region of $\Sigma$. Writing $n^{\alpha}$ as the normal to $u^{\alpha}$ in the tangent space of $\Sigma$ we restrict $\mathcal{M}$ by the condition that there exists $u^{\alpha}$ such that

$$
G_{\alpha}^{\beta} u^{\alpha} n_{\beta}=0,
$$

where $G_{\alpha}^{\beta}$ is the Einstein tensor of $\mathcal{M}$. (The Vaidya metric [4] (corresponding to a null flux) provides a familiar example of an $\mathcal{M}$ for which such a flow does not exist.) Now $u^{\alpha}$ (and $n^{\alpha}$ ) are, if they exist, uniquely determined from the metric alone. (Equations $u^{\alpha} u_{\alpha}=-1, n^{\alpha} n_{\alpha}=1, u^{\alpha} n_{\alpha}=0$ and the zero flux condition (2) are solved for the components of $u^{\alpha}$ and $n^{\alpha}$ and the appropriate roots determined by the time orientation of $\mathcal{M}$ (say $u^{1}>0$ ).) The algorithm for producing $u^{\alpha}$ for specific coordinates on $\Sigma$ is straightforward and discussed elsewhere in a more general context [5]. In what follows we make use of the following scalars:

$$
G \equiv G_{\alpha}^{\alpha}, G 1 \equiv G_{\alpha}^{\beta} u^{\alpha} u_{\beta}, G 2 \equiv G_{\alpha}^{\beta} n^{\alpha} n_{\beta}, \Delta \equiv \sigma_{\alpha}^{\beta} n^{\alpha} n_{\beta}, \Psi \equiv G+G 1-3 G 2, \mathcal{W} \equiv C_{\alpha \beta \gamma \delta} C^{\alpha \beta \gamma \delta},
$$

where $\sigma_{\alpha}^{\beta}$ is the shear associated with $u^{\alpha}$ and $C_{\alpha \beta \gamma \delta}$ is the Weyl tensor. A necessary and sufficient condition for the conformal flatness of $\mathcal{M}$ is $\mathcal{W}=0$.

Perhaps the most important function associated with $\mathcal{M}$ is the effective gravitational mass $M\left(x^{1}, x^{2}\right)$ the invariant properties of which were first explored by Hernandez and Misner [6] who wrote the function in the form

$$
M=\frac{R^{3}}{2} \mathcal{R}_{\theta \phi}{ }^{\theta \phi},
$$

where $\mathcal{R}$ is the Riemann tensor of $\mathcal{M}$. See also [7], [8], [9] and [10] for further discussion and [1] for a recent application. In terms of the scalars (3) $M$ can be given as

$$
M=\frac{R^{3}}{12}(G+3(G 1-G 2)+\sqrt{3 \mathcal{W}})
$$

from which we immediately obtain the following necessary and sufficient condition for the conformal flatness of $\mathcal{M}$ :

$$
M=\frac{R^{3}}{12}(G+3(G 1-G 2)) .
$$


From (2) and (5) it follows that

$$
M^{\prime}=\frac{G 1}{2} R^{2} R^{\prime}
$$

where' is the intrinsic derivative in the direction $n^{\alpha}$. (For the case of a perfect fluid in comoving coordinates (7) is well known (e.g. 2] section 1.4).) Assuming that $\mathcal{M}$ is non-singular (the scalars (3) remain finite) and has an origin $(R=0)$ it follows from (5) and (7) that

$$
G 1^{\prime}=0 \Leftrightarrow G+G 1-3 G 2+\sqrt{3 \mathcal{W}}=0
$$

or, equivalently,

$$
G 1^{\prime}=0 \Leftrightarrow M=\frac{G 1 R^{3}}{6} .
$$

Observe that if

$$
\Psi=0
$$

then

$$
G 1^{\prime}=0 \Leftrightarrow \mathcal{W}=0
$$

To transform the forgoing invariant geometrical statements into physical phenomenological parameters by way of Einstein's equations, decompose the energy-momentum tensor in the form

$$
T_{\beta}^{\alpha}=\rho u^{\alpha} u_{\beta}+p_{1} n^{\alpha} n_{\beta}+p_{2} \delta_{\beta}^{\alpha}+p_{2}\left(u^{\alpha} u_{\beta}-n^{\alpha} n_{\beta}\right)-2 \eta \sigma_{\beta}^{\alpha} .
$$

Einstein's equations are effectively reversed by way of the following observation: Substitution of

$$
\begin{gathered}
\rho=\frac{G 1}{8 \pi}, \\
p_{1}=\frac{G 2}{8 \pi}+2 \eta \Delta,
\end{gathered}
$$

and

$$
p_{2}=\frac{G+G 1-G 2}{16 \pi}-\eta \Delta,
$$

into (12) and multiplication by $8 \pi$ produces $G_{\alpha}^{\beta}$. Note in particular that there are at most three independent Ricci scalars in this case and that the syzygy (17) of [12] for the decomposition (12) must, by way of the foregoing inversion, reduce to an identity as can be directly verified [5]. The following specializations arise, where in all cases the energy density is given by (13):

a. $\quad \eta \Delta=0: \quad$ If $\eta \Delta=0$ (one or more of $\eta, \Delta$ or $\sigma_{\alpha}^{\beta}=0$ ) then $\Psi=0$ is a necessary and sufficient condition for a perfect fluid $\left(p_{1}=p_{2}, \eta=0\right)$.

b. $p_{1} \equiv p_{2} \equiv p, \Delta \neq 0: \quad$ If $p_{1} \equiv p_{2} \equiv p, \Delta \neq 0$ it follows from (14) and (15) that

$$
p=\frac{G+G 1}{24 \pi},
$$

and

$$
\eta=\frac{G+G 1-3 G 2}{48 \pi \Delta},
$$

so that $\Psi=0$ is once again a necessary and sufficient condition for a perfect fluid. 
c. $p_{1} \neq p_{2}, \Delta \neq 0$ : Now $p_{1}$ is given by (14) and $p_{2}$ by (15) where $\eta$ is a freely specified function. The special choice $\eta \equiv 0$ reduces to case a.

Clearly any spacetime $\mathcal{M}$ satisfying (2) is an "exact solution" of Einstein's equations: simply put (13), (14) and (15) into (12). Only with the imposition of a constraint, like $\Psi=0$, is $\mathcal{M}$ restricted. For example, in the static case, where $\sigma_{\alpha}^{\beta}=0$, any $\mathcal{M}$ is a "solution", but with the constraint $\Psi=0$ relatively few available solutions make any physical sense [13] 14]. As emphasized previously, only those "exact solutions" which make physical sense are worthy of consideration. The possibility that a given $\mathcal{M}$ might have more than one physically reasonable interpretation is not excluded, but all possible interpretations subject to (12) are delineated above. Because the foregoing procedure is algorithmic, and because alternate interpretations of know solutions are of some interest if physically justified, the procedure is being incorporated into the interactive database GRDB [15].

In terms of the parameters $\left(\rho, p_{1}, p_{2}, \eta\right)$ we have

$$
\begin{gathered}
M=\frac{R^{3}}{12}\left(16 \pi\left(\rho+p_{2}-p_{1}+3 \eta \Delta\right)+\sqrt{3 \mathcal{W}}\right), \\
\mathcal{W}=0 \Leftrightarrow M=\frac{4 \pi}{3} R^{3}\left(\rho+p_{2}-p_{1}+3 \eta \Delta\right), \\
G 1^{\prime}=0 \Leftrightarrow M=\frac{4 \pi}{3} R^{3} \rho,
\end{gathered}
$$

and if

$$
p_{2}-p_{1}+3 \eta \Delta=0
$$

then

$$
G 1^{\prime}=0 \Leftrightarrow \mathcal{W}=0
$$

Condition (22) generalizes a previously known condition [16]. A perfect fluid is a special case of (21) and in that case we have

$$
G 1^{\prime}=0 \Leftrightarrow \mathcal{W}=0 \Rightarrow \sigma_{\beta}^{\alpha}=0
$$

where the last implication comes from the fact that all conformally flat perfect fluids are shear free 17]. The condition $G 1^{\prime}=0 \Rightarrow \sigma_{\beta}^{\alpha}=0$, for a perfect fluid in the comoving frame, is stated in [1] (section (14.2.4)) and attributed to [18] and [19. Neither [1] (in that section), 18] nor [19] even mention the Weyl tensor, the vanishing of which, as the foregoing makes clear, can be considered central to the result.

\section{Acknowledgments}

This work was supported by a grant from the Natural Sciences and Engineering Research Council of Canada. Portions of this work were made possible by use of GRTensorII [20]. It is a pleasure to thank Don Page and Nicos Pelavas for comments. A more complete account of related work, but with a different emphasis, is in progress with Mustapha Ishak.

[*] Electronic Address: lake@astro.queensu.ca

[1] D. Kramer, H. Stephani, E. Herlt and M. MacCallum, Exact Solutions of Einstein's Field Equations (Cambridge University Press, Cambridge, 1980).

[2] A. Krasinski, Inhomogeneous Cosmological Models (Cambridge University Press, Cambridge, 1997).

[3] We use signature +2 with $\alpha=1, \ldots, 4$.

[4] P. C. Vaidya, Proc. Indian Acad. Sci. A33, 264 (1951).

[5] M. Ishak and K. Lake (2003) (in preparation) 
[6] W. C. Hernandez and C. W. Misner, Astrophys. J. 143, 452 (1966).

[7] M. E. Cahill and G. C. McVittie, J. Math. Phys, 11, 1360 (1970).

[8] E. Poisson and W. Israel, Phys. Rev D 41, 1796 (1990).

[9] T. Zannias, Phys. Rev. D 41, 3252 (1990)

[10] S. Hayward, Phys. Rev. D 53, 1938 (1996) (gr-qc/9408002).

[11] S. Rahman and M. Visser, Class. Quant. Grav. 19935 (2002) (gr-qc/0103065).

[12] D. Pollney, N. Pelavas, P. Musgrave and K. Lake, Computer Physics Communications 115, 381 (1998)(gr-qc/9809012).

[13] M. S. R. Delgaty and K. Lake, Computer Physics Communications 115, 395 (1998) (gr-qc/9809013).

[14] N. Neary, J. Lattimer and K. Lake (in preparation).

[15] M. Ishak and K. Lake, Class. Quantum Grav. 19, 505 (2002) (gr-qc/0111008) (http://grdb.org)

[16] K. Lake, Phys. Rev D 40, 1344 (1989) (D 40, 4201).

[17] H. Stephani, Commun. Math. Phys. 5, 337 (1967).

[18] I. H. Thompson and G. J. Whitrow, Mon. Not. R. astr. Soc. 136, 207 (1967).

[19] R. M. Misra and D. C. Strivastava, Phys. Rev. D 8, 1653 (1973).

[20] This is a package which runs within Maple. It is entirely distinct from packages distributed with Maple and must be obtained independently. The GRTensorII software and documentation is distributed freely on the World-Wide-Web from the address http://grtensor.org 